\title{
La agenda europea de prevención de conflictos veinte años después del Programa de Gotemburgo
}

\section{The European conflict prevention agenda twenty years after the Gothenburg Program}

JARA CUADRADO

Universidad Autónoma de Madrid y OBSERVARE (Universidad Autónoma de Lisboa) Departamentode Historia Contemporánea. Facultad de Filosofía y Letras. Campus de Cantoblanco. C/Francisco Tomás y Valiente, 1. 28049

jaracb.4488@gmail.com

(c) $\frac{(1)(9)}{\text { Ev }}$ Este artículo está sujeto a una licencia "Creative Commons ReconocimientoNo Comercial" (CC-BY-NC).

DOI: https://doi.org/10.24197/ree.79.2022.1-28

Resumen: La prevención de conflictos se ha posicionado, desde los años noventa, como una doctrina clave en la gestión de crisis. La Unión Europea (UE) la incorporó a su agenda de acción exterior en aquel momento $y$, desde entonces, ha pasado por diferentes etapas, tratando de adaptarse al contexto internacional de seguridad, cada vez más complejo y cambiante. Esa evolución que ha experimentado la política europea de prevención de conflictos es el objeto de estudio de este artículo, con el propósito de conocer los cambios en la doctrina y cómo estos han repercutido en un elemento fundamental para el éxito de la prevención: la cooperación con los actores locales y regionales del contexto conflictual en el que se interviene. Con la finalidad de conocer mejor el potencial de esa relación, se ha seleccionado el caso de la Comunidad Económica de los Estados de África Occidental (CEDEAO), debido a las importantes relaciones que mantiene con la UE, a su experiencia en el campo de la prevención y por la relevancia estratégica que esa zona tiene para Europa.

Palabras clave: Unión Europea, gestión de crisis, prevención de conflictos, África, CEDEAO.

Abstract: Conflict prevention has been a key doctrine in crisis management since the nineties. The European Union (EU) incorporated it into its foreign action agenda at that time and, since then, it has gone through different stages, trying to adapt to the increasingly complex and changing international security context. The evolution the European conflict prevention policy has undergone is the object of study of this article, with the purpose of knowing the changes in doctrine and how these have had an impact on a fundamental element for the success of prevention: cooperation with the local and regional actors of the conflictual context in which the intervention occurs. In order to better understand the potential of this relationship, the case of the Economic Community of West African States (CEDEAO) has been selected, due to the important relations it maintains with the EU, its experience in the field of prevention and, therefore, the strategic relevance that this area has for Europe.

Keywords: European Union, crisis management, conflict prevention, Africa, ECOWAS. 


\section{INTRODUCCIÓN}

El espíritu que siempre ha acompañado a la Unión Europea (UE) desde que se pusieron en pie los primeros cimientos comunitarios ha respondido al fin último de preservar la paz y estabilidad entre los diferentes pueblos.

El proyecto europeo ha evolucionado y se ha ido adaptando a las necesidades requeridas por las transformaciones experimentadas en la escena internacional, así como a los retos y desafíos internos. La UE despedía el siglo pasado siendo uno de los principales actores globales. En materia de paz y seguridad, se ha convertido en una pieza fundamental de numerosos procesos (desde misiones de ayuda humanitaria, a labores de entrenamiento de las fuerzas de seguridad de terceros países, programas de cooperación al desarrollo, u observación electoral), como parte de su Política Exterior y de Seguridad Común (PESC), evidenciando sus fortalezas pero también visibilizando sus debilidades.

Aunque el número de conflictos armados ha vivido un importante descenso a partir de los años noventa (Newman, 2009), las cifras podrían haberse recuperado en los últimos años (Center for Systemic Peace, 2019). En la actualidad, los escenarios de crisis internacionales presentan una naturaleza multidimensional, donde convive un amplio abanico de amenazas securitarias en su sentido más amplio (Marc, Verjee y Mogaka, 2015; Cuadrado, 2018). La comunidad internacional -siendo la UE uno de los actores más relevantes-, ha respondido a este contexto bañado por la globalización con un amplio conjunto de políticas de gestión de crisis adaptadas a una realidad rápidamente cambiante. Fue a principios de este siglo xxi cuando la agenda de prevención de conflictos empezó a desarrollarse de forma oficial, tras unos años noventa caracterizados por desastres humanitarios que dejaron una importante huella en el listado de errores y fracasos de los principales actores políticos.

Ante ese marco, se impulsa el Programa Gotemburgo para la Prevención de Conflictos Violentos, aprobado durante una reunión del Consejo Europeo los días 15 y 16 de junio de 2001 por iniciativa de la presidencia sueca. Nace con el propósito de impulsar la prevención de conflictos como uno de los principales objetivos de la política exterior de 
la UE y de contribuir a una cultura global de la prevención ${ }^{1}$. Con la puesta en marcha del Programa de Gotemburgo, se abría una gran oportunidad para la UE para liderar, en la escena internacional, la gestión de crisis desde una perspectiva preventiva, aspirando a superar el tradicional enfoque reactivo que había caracterizado la agenda hasta entonces.

A partir de ese momento, la prevención de conflictos ha ido ganando espacio en la documentación oficial de la UE, desarrollando diversas iniciativas en esta dirección. Esta política ha vivido diferentes etapas, respondiendo al contexto internacional y a los propios avatares internos de la organización. Los acontecimientos de la última década, bañada por la crisis económica y, posteriormente, por la crisis de refugiados, así como los retos más próximos, como la crisis desencadenada por la pandemia, han condicionado la agenda exterior europea. El escenario reciente, con una posible vuelta al multilateralismo -muy esperada en los principales foros europeos-, podría abrir una oportunidad y crear un camino para relanzar una agenda de prevención. Son varias las ocasiones recientes en las que instituciones europeas, como el Parlamento ${ }^{2}$, han subrayado la necesidad de impulsar la prevención de conflictos como pieza fundamental para hacer frente a los actuales retos de seguridad ${ }^{3}$.

La UE tiene una posición, podría decirse, privilegiada, para convertirse en un gran actor global en el campo de la prevención de conflictos. No se trata solamente de impulsar estas políticas por una motivación de carácter moral y/o humanitario -pues la experiencia ha demostrado que la prevención puede salvar más vidas que aquellas medidas que se llevan a cabo una vez que la violencia ha estallado 4 - sino que existen unos intereses estratégicos, económico-comerciales y securitarios que pueden incentivar a los Estados Miembros para apostar

\footnotetext{
${ }^{1}$ Informe sobre las Conclusiones del Consejo Europeo de Gotemburgo celebrado los días 15 y 16 de junio de 2001 (SN 200/1/01 REV 1).

${ }^{2}$ Informe del Parlamento Europeo del 10 de julio de 2018, Reforzar las capacidades de la Unión en la prevención de conflicto y mediación (2018/2159(INI)).

${ }^{3}$ Resolución del Parlamento Europeo del 12 de marzo de 2019 sobre la creación de capacidad de la Unión en materia de prevención de conflictos y mediación (2018/2159(INI)).

${ }^{4}$ Así se subrayaba, por ejemplo, en el Informe Brahimi (2000), liderado por el embajador argelino, Lakhdar Brahimi tras la petición del entonces Secretario General de Naciones Unidas, Kofi Annan, donde se subrayaba que la prevención "es menos costosa para la comunidad internacional que una acción militar, que la asistencia humanitaria de emergencia o que la reconstrucción después de la guerra”.
} 
por una agenda enfocada en la prevención. Es, además, un camino más económico y duradero a largo plazo que la opción de trabajar sobre las consecuencias provocadas por el estallido de un conflicto, tal y como aparece en la página del Servicio Europeo de Acción Exterior (EEAS por sus siglas en inglés $)^{5}$ : "fallar en la prevención de conflictos [...] revierte años de desarrollo y aumenta la necesidad de [implementar] reacciones costosas".

Bajo este contexto, el objeto de estudio de este artículo es analizar la evolución de la agenda de prevención de conflictos de la UE desde la aprobación del Programa de Gotemburgo, en 2001, hasta la actualidad, para entender la postura europea en el escenario internacional en el marco de la gestión de crisis, así como el potencial que tiene para superar la cultura de reacción. En este sentido, nos preguntamos, en primer lugar, si la UE ha desarrollado una política de prevención eficaz y, en segundo lugar, cuáles han sido los factores que han condicionado dicha política.

Con el fin de evaluar estos aspectos, pero también para conocer la ventana de posibilidades que se abre en esta dirección, se centrará la atención en un elemento clave, subrayado de forma recurrente en la documentación europea, pero sobre el que no se ha trabajado lo suficiente; la cooperación con terceros actores para implementar una política de prevención de conflictos más comprehensiva y eficaz. Se ha seleccionado como estudio de caso la región de África occidental y su actor regional, la Comunidad Económica de los Estados de África Occidental (CEDEAO), por dos motivos: (1) por tratarse de un área geográfica de especial relevancia e interés para la UE y (2) debido a que este actor regional ha liderado una agenda preventiva de gran relevancia dentro del continente africano. En este sentido, y dada la importancia que África occidental tiene para la UE por diversos motivos (Marc, Verjee y Mogaka, 2015) -relaciones comerciales, diplomáticas, vínculos culturales e históricos-, siendo una de las zonas en las que más misiones ha desplegado, es necesario entender y ampliar el conocimiento acerca de las ventajas y obstáculos que encierra un trabajo conjunto en materia de prevención de conflictos entre ambos actores políticos.

La estructura del artículo es la siguiente. Tras una introducción donde se presenta el objeto de estudio, se desarrolla la agenda de prevención de conflictos de la UE desde el año 2001, analizando los

\footnotetext{
${ }^{5}$ Accesible en el siguiente enlace: https://eeas.europa.eu/topics/security-defence-crisisresponse/426/conflict-prevention-peace-building-and-mediation en
} 
pasos dados en este sentido y los distintos instrumentos o herramientas con las que cuenta la Unión para intervenir de forma preventiva. A continuación, se expone la importancia de desarrollar un trabajo de cooperación con los actores que tienen una implicación directa sobre el terreno, más próximos al contexto de crisis y/o conflicto, elemento clave para garantizar una política de prevención de conflictos eficaz y duradera a largo plazo. Para ello, se analiza el caso de la CEDEAO, presentando brevemente su política de prevención de conflictos para, posteriormente, evaluar las ventajas que ofrece a la UE, así como identificar algunos de los obstáculos detectados en la práctica, que dificultan que la agenda preventiva sea implementada con éxito. Finalmente, se desarrollan unas conclusiones acerca del papel de la UE en la prevención de conflictos y la agenda de futuro que se presenta, especialmente en materia de cooperación con terceros actores.

El análisis se apoya en documentación oficial de los órganos europeos y la CEDEAO, así como en monografías y artículos especializados e informes sobre la actividad de ambas organizaciones sobre el terreno.

\section{Evolución de LA DOCTRINA EUROPEA DE PREVENCIÓN DE CONFLICTOS}

El concepto de prevención de conflictos puede entenderse como el conjunto de medidas destinadas a evitar que situaciones de violencia e inseguridad se conviertan en un conflicto armado, minimizar y contener tensiones manifiestas, así como transformar contextos en los que subyacen factores que atentan contra la seguridad de las personas en procesos pacíficos y estables "de cambio político y social" (Ramsbotham, Miall \& Woodhouse, 2011, p.113). Aunque la prevención se aplica especialmente al comienzo del ciclo del conflicto (Lund, $1993)^{6}$, cuando las tensiones y disputas están en proceso de convertirse en conflictos violentos, esta debe estar presente durante toda la vida del conflicto. Los esfuerzos deben centrarse no solo en intentar contener o prevenir una situación de violencia armada, sino también en la resolución

\footnotetext{
${ }^{6}$ La teoría de la curva del conflicto, desarrollada por Michael Lund en los años noventa, hace referencia a las distintas fases de vida de un conflicto, desde la situación previa (pre-conflicto), pasando por las fases de conflicto violento y guerra, para después descender hacia la etapa de recuperación posconflicto.
} 
de los factores que conducen a esa situación. Teniendo esta perspectiva presente, la prevención puede ser utilizada de forma inmediata, ante una crisis inminente (prevención operativa) o puede ponerse en marcha a través de medidas que funcionan a largo plazo (prevención estructural) (Carnegie Corporation of New York, 1997). Esta visión integral de la prevención de conflictos es la que subyace en la documentación oficial de la UE.

La Unión Europea es un ejemplo fundamental en el desarrollo de políticas preventivas, pues la propia organización se constituyó con tal objetivo; el de la prevención de conflictos violentos en su territorio. En el desarrollo de su PESC, se han elaborado medidas destinadas a la gestión y prevención de conflictos, motivadas estas por la aspiración de crear un entorno de paz y estabilidad.

Las lecciones obtenidas de Yugoslavia sirvieron para dar mayor valor a la prevención de conflictos, no sólo en suelo europeo, sino también en los entornos de interés estratégico de la Unión, y se vio la necesidad de desarrollar mecanismos e instrumentos para estar preparados ante situaciones como aquella.

Los inicios de la organización europea en el ámbito de la prevención de conflictos estuvieron vinculados a aspectos más estructurales, como las políticas de desarrollo, que han estado siempre lideradas a nivel mundial por la UE. Como ha señalado Stewart (2006), el progreso de la UE en la prevención de conflictos se produjo principalmente a mediados de la década de 1990 en el ámbito del desarrollo y aplicado al continente africano. En esos momentos, la UE centraba sus esfuerzos en aspectos como "el fortalecimiento del estado de derecho, el apoyo a las instituciones democráticas, el desarrollo de la sociedad civil y la reforma del sector de la seguridad" (Wouters y Naert, 2003, p.27).

Sin embargo, a fines del siglo xx, la organización comenzó a incorporar medidas más operativas y directas, perdiendo su asociación exclusiva con la esfera del desarrollo (Stewart, 2006). Así, como parte de su PESC, asumió otro tipo de actividades que empezar a transcender la esfera de la cooperación y el desarrollo. El Tratado de Ámsterdam de 1997 propuso, por ejemplo, el establecimiento de una unidad de planificación de políticas y alerta temprana, que tendrá, entre otras tareas, el objetivo de proporcionar "evaluaciones y alerta rápida en cuanto a los hechos o situaciones que pudieran tener repercusiones importantes para 
la política exterior y de seguridad de la Unión, incluidas las crisis políticas potenciales", .

La prevención de conflictos va ganando espacio entre los documentos de la UE. En el Informe de 1992 del Consejo Europeo de Lisboa, se destacó la necesidad de fortalecer la prevención de conflictos:

la PESC debe contribuir a garantizar que la acción exterior de la Unión sea menos reactiva a los acontecimientos del mundo exterior y más activa en la [...] creación de un entorno internacional más favorable. Esto permitirá a la Unión Europea tener una capacidad mejorada para abordar los problemas en sus raíces con el fin de anticipar el estallido de crisis ${ }^{8}$.

En 1996 se produce una mención a la prevención de conflictos en uno de los documentos oficiales, la Comunicación de la Comisión Europea sobre la prevención de conflictos en África ${ }^{9}$. En el Informe Improving the Coherence and Effectiveness of the European Union Action in the Field of Conflict Prevention, se subraya que "la prevención de conflictos está en el corazón de la Unión Europea, que es en sí misma un ejemplo exitoso de cómo se pueden promover la reconciliación, la estabilidad y la prosperidad mediante cooperación y entendimiento más estrechos"10.

Un punto de inflexión importante se produce en el año $2001^{11}$, cuando se aprueban dos documentos clave: la Comunicación de la Comisión Europea sobre la Prevención de Conflictos y, especialmente, el Programa de Gotemburgo para la Prevención de Conflictos Violentos, adoptado por el Consejo Europeo. Ambos pasos evidencian el

\footnotetext{
${ }^{7}$ Tratado de Ámsterdam por el que se modifican el Tratado de la Unión Europea, los Tratados Constitutivos de las Comunidades Europeas y determinados actos conexos, del 10 de noviembre de 1997 (97/C 340/01): Declaración sobre el establecimiento de una Unidad de planificación de la política y de alerta rápida (2c).

${ }^{8}$ Anexo I de las Conclusiones del Consejo Europeo de Lisboa, 26-27 de junio de 1992 (Bull EC 6-1992), para. I.31.

${ }^{9}$ Comunicación de la Comisión Europea del 6 de marzo 1996 sobre la prevención de conflictos en África (SEC (96) 332).

${ }^{10}$ Informe presentado al Consejo Europeo de Niza por el Secretario General/Alto Representante y la Comisión, 7-9 de diciembre de 2000, Improving the Coherence and Effectiveness of the European Union Action in the Field of Conflict Prevention $(033 / 00)$, I.1.

${ }^{11}$ Se trata del mismo año en el que el Secretario General de las Naciones Unidas publica su primer informe sobre la prevención de conflictos.
} 
compromiso que la UE asumía con la agenda de prevención de conflictos.

Las líneas del Programa apuntaban a: establecer prioridades políticas claras para acciones de prevención; mejorar la alerta rápida, la reacción temprana y la coherencia política; fomentar instrumentos de prevención a largo y corto plazo; y a la cooperación internacional con socios de la $\mathrm{UE}^{12}$.

En la Comunicación, se diferencia entre prevención estructural y operativa, ya que se subraya que la UE tiene a su disposición una amplia gama de instrumentos para la acción a largo o corto plazo ${ }^{13}$. Dentro del primer grupo, el documento subraya la política de desarrollo y otros programas de cooperación, instrumentos de política comercial, apoyo a la democracia, estado de derecho, sociedad civil, medios de comunicación independientes, igualdad de género o reforma del sector de la seguridad. A pesar de que se nombran explícitamente por primera vez aquellas medidas que la UE consideraba herramientas a corto plazo -Mecanismo de Reacción Rápida, diálogo político y mediación, Representantes Especiales de la UE, sanciones económicas o de otro tipo, o seguimiento de acuerdos internacionales-, citadas como aquellas para "reaccionar rápidamente a los conflictos nacientes", lo cierto es que se evidencia que aún estaban poco desarrolladas en ese momento, ya que gran parte del texto estaba dedicado a medidas preventivas a largo plazo.

Desde la aprobación del Programa de Gotemburgo, la Comisión Europea comenzó a dar más relevancia a la prevención de conflictos y a desarrollar diferentes iniciativas, incluyéndola en los tratados de gobierno y en diferentes documentos políticos clave. La prevención de conflictos se menciona en la Estrategia Europea de Seguridad. Una Europa segura en un mundo mejor, del año 2003, donde se destaca la necesidad de:

"actuar antes de que la situación en los países a nuestro alrededor se deteriore, cuando se detecten señales de proliferación, y antes de que se produzcan emergencias de orden humanitario. La intervención preventiva puede evitar que se planteen problemas más graves en el futuro"14.

12 Borrador del Consejo Europeo sobre el Programa de la Unión Europea para la Prevención Violentos, del 7 de junio de 2001 (9537/1/01 REV 1), 6.

${ }^{13}$ Comunicación de la Comisión Europea del 11 de abril de 2001 sobre prevención de conflictos (COM(2001) 211 final), p.4.

${ }^{14}$ Consejo de la Unión Europea, Estrategia Europea de Seguridad. Una Europa segura en un mundo mejor, 2003, p.40. Disponible en: https://www.consilium.europa.eu/media/30808/qc7809568esc.pdf 
Como señala Niño Pérez (2006, p.131), es en el Tratado de Constitución Europea aprobado en el año 2004, "donde se encuentra por primera vez en el campo normativo de la Unión Europea una referencia explícita a la prevención de conflictos como uno de los objetivos de la acción exterior de la Unión Europea", pues hasta ahora se trataba de informes.

En el Tratado de Lisboa (2007) ${ }^{15}$, se refuerza esta doctrina, incorporando de forma clara la prevención de conflictos como una de las misiones principales de su política exterior, ya que defiende que la UE tiene como objetivo "preservar la paz, prevenir conflictos y fortalecer la seguridad internacional"16. A través del Tratado de Lisboa, se otorga a los Estados Miembros un mandato para comprometerse con la prevención de conflictos (Juncos y Blockmans, 2018). En este documento jurídico se recuerda que la Unión tiene "capacidad operativa respaldada por medios civiles y militares [...para] garantizar el mantenimiento de la paz, la prevención de conflictos y el refuerzo de la seguridad internacional" ${ }^{17}$. Así, las medidas operativas, como parte de su PESC, cobraron una mayor relevancia.

La primera década de vida del Programa de Gotemburgo, un plan específico de la UE para impulsar su papel en la prevención de conflictos, estuvo acompañada por la aprobación de diferentes documentos doctrinarios que incluían la prevención de conflictos como un eje principal sobre el que articular la política exterior de la $\mathrm{UE}^{18}$.

El 2011 fue un año clave, pues se aprobaron los documentos Conclusiones del Consejo de la Unión Europea sobre prevención de conflictos y el Informe de la Comisión Europea Thematic Evaluation of European Commission Support to Conflict Prevention and

\footnotetext{
${ }^{15}$ El Tratado de Lisboa, por el que se modifican el Tratado de la Unión Europea y el Tratado constitutivo de la Comunidad Europea (DO C 306 de 17.12.2007), fue aprobado el 13 de diciembre de 2007, con entrada en vigor el 1 de diciembre de 2009.

${ }^{16}$ Ibidem, artículo 21(2).

${ }^{17}$ Ibidem, artículo 21(c).

${ }^{18}$ Algunos documentos significativos fueron el Concepto sobre el fortalecimiento de las capacidades de mediación y diálogo de la UE, de 10 de noviembre de 2009 (15779/09), actualizado en el año 2020 (EU Mediation Concept in 2020), donde se expone el papel de la UE para impulsar su diplomacia preventiva; o los informes Towards a UE Response to Situations of Fragility, del 25 de octubre de 2008, Implementation of the European Security Strategy. Providing Security in a Changing World, del 11 de diciembre de 2008, o EU Strategic Framework and Action Plan on Human Rights and Democracy, del 25 de junio de 2012.
} 
Peacebuilding. El primero se presenta como una declaración de la organización europea para seguir apostando por la prevención de conflictos $^{19}$. Aunque el documento se recibió como una declaración clave en la evolución de la agenda de prevención, lo cierto es que no fue más que un resumen de aquellos instrumentos con los que contaba la UE para impulsar la prevención, sin aportar un análisis en profundidad sobre dicha agenda. Ese análisis sí se lleva a cabo en el segundo documento, donde se destacan algunos déficits, como, por ejemplo, el trabajo cooperativo, subrayando la "debilidad en cuanto a la sinergia entre los niveles local, nacional y regional", limitada esa colaboración a aspectos como "el intercambio de información" 20 .

A pesar de apostar por un enfoque integral y defender una política preventiva a corto y largo plazo, lo cierto es que la experiencia hasta ese momento demostraba que el término 'prevención de conflictos' se ha había entendido principalmente como aquel destinado a desplegar medidas que se desarrollan especialmente a largo plazo, con una ausencia importante en la doctrina sobre la implementación de aquellas medidas a corto plazo.

El inicio de la siguiente década estuvo marcado por las consecuencias de la crisis económica que estalla a partir de 2007, con fuertes repercusiones en la agenda exterior de la UE, las llamadas 'primaveras árabes' y su onda expansiva, hasta llegar a la guerra civil siria y la crisis de refugiados. Estos hechos impactan en la Estrategia de Seguridad de 2016, Una visión común, una actuación conjunta: una Europa más fuerte. Estrategia global para la politica exterior y de seguridad de la Unión Europea, y la orientación que se da a la política preventiva:

Es esencial aplicar un enfoque pluridimensional mediante la utilización de todos los instrumentos y políticas disponibles orientados a la prevención, la gestión y la resolución de conflictos [...] No existen soluciones rápidas para ninguno de estos conflictos [...] Redoblaremos por tanto nuestros esfuerzos en materia de prevención, vigilando las causas fundamentales de los conflictos, como las violaciones de los

${ }^{19}$ Conclusiones del Consejo de la Unión Europea del 20 de junio de 2011 sobre prevención de conflictos.

${ }^{20}$ Informe Final de Comisión Europea de octubre de 2011 Thematic Evaluation of European Commission Support to Conflict Prevention and Peacebuilding, volumen 1, p.iii. 
derechos humanos, la desigualdad, la tensión derivada de la escasez de recursos naturales, y el cambio climático ${ }^{21}$.

En un documento posterior, The European Union's Global Strategy. Three Years On, Looking Forward, se muestra un concepto amplio de prevención de conflictos, ya que considera que aspectos como invertir "en seguridad alimentaria" o "acción climática" forman parte de las políticas de prevención de conflictos ${ }^{22}$. Más reciente es la Estrategia de la UE para una Unión de la Seguridad para el período 2020-2025 presentada el 24 de julio de 2020 por medio de una comunicación de la Comisión Europea y cuyos objetivos se han ido conociendo a través de una serie de informes y comunicados de la Comisión ${ }^{23}$. En el primer comunicado se subraya la necesidad de "desarrollar capacidades y medios para la detección temprana y la respuesta rápida a las crisis de seguridad mediante un enfoque integrado y coordinado" ${ }^{24}$, aunque más bien este enfoque preventivo se dirige especialmente a amenazas como la radicalización y el terrorismo o la ciberdelincuencia. Sin embargo, en lo que se refiere a la gestión de crisis externas, apenas existen referencias a medidas directas, existiendo una falta de concretización sobre las opciones y herramientas de las que dispone la UE para hacer efectiva esa voluntad preventiva. Como apunta Elena Baracani (2016, p.228), la UE "prefiere llevar a cabo la prevención de conflictos estructural a través de herramientas de política exterior no convencionales, como la asistencia económica, el diálogo político estructurado y la cooperación regional, para tratar de cambiar las condiciones socioeconómicas que subyacen al conflicto" antes que otras medidas de tipo operativo, incluyendo la amenaza del uso de la fuerza, una vez que la violencia ha escalado, y en las que la organización no se encuentra cómoda.

A pesar de esta carencia, se puede decir que la UE considera que las medidas operativas también deben formar parte de su abanico de

${ }^{21}$ Estrategia Europea de Seguridad. Una visión común, una actuación conjunta: una Europa más fuerte. Estrategia global para la política exterior y de seguridad de la Unión Europea, 2016, p.23.

${ }^{22}$ The European Union's Global Strategy. Three Years On Looking. Disponible en: https://eeas.europa.eu/sites/default/files/eu_global_strategy_2019.pdf

${ }^{23}$ Disponibles en el siguiente enlace: $\mathrm{https} / / / \mathrm{ec}$.europa.eu/info/strategy/priorities-20192024/promoting-our-european-way-life/european-security-union es.

${ }^{24}$ Comunicación de la Comisión al Parlamento Europeo, al Consejo Europeo, al Consejo, al Comité Económico y Social Europeo y al Comité de las Regiones del 24 de julio de 2020 sobre la Estrategia de la UE para una Unión de la Seguridad (COM(2020) 605 final), p.6. 
acciones para alcanzar la prevención de conflictos, ya que diferentes documentos apuntan en ese sentido ${ }^{25}$. La Estrategia de Seguridad de 2016, por ejemplo, recuerda que la UE tiene que "actuar en todas las fases del ciclo del conflicto",26.

Así, parece ser que la organización apuesta, al menos en la teoría, por un enfoque integral en la prevención de conflictos que incluye medidas tanto a largo como a corto plazo, y diferencia este tipo de medidas de la gestión de crisis o la construcción de paz.

El desarrollo de estas políticas se ha impulsado a través de la construcción de diferentes órganos y estructuras desde las que poner en marcha una agenda encaminada hacia la prevención. La creación en 2011 del Servicio Europeo de Acción Exterior, que cuenta con un área dedicada a la Prevención de Conflictos, la Construcción de Paz y la Mediación, ha sido clave. Desde aquí se coordinan algunos instrumentos y estructuras que abordan, desde un enfoque integral, la prevención de conflictos como parte de la PESC. El Instrument for Stability (IfS), creado en 2007 y que sustituyó al Rapid Reaction Mechanism, cubre actividades tanto a largo como a corto plazo, desde la mediación y el diálogo, los procesos electorales, o "el apoyo a los componentes civiles de las misiones integradas de mantenimiento de la paz, así como estabilización y misiones de seguimiento" (Schünemann, 2011, p.2). En 2017 se crea PRISM, la sección de Prevención de conflictos, Estado de derecho/RSS, Enfoque integrado, Estabilización y Mediación y, más recientemente, el EEAS ha impulsado la Pool of EU Peace Mediators, para fortalecer el alcance de la mediación de la UE y capacidad operacional. Otras estructuras que juegan un papel destacado son el sistema de alerta temprana (EWS) y los Regional Crisis Response Planning Officers (RCRPOS), el EEAS Mediation Support Team o el EU Crisis Response Mechanism. Estos son solo algunos de los ejemplos que muestran que la UE dispone de una estructura amplia para la toma de decisiones a la hora de abordar la prevención de conflictos de forma inmediata.

\footnotetext{
${ }^{25}$ Comunicación conjunta al Parlamento Europeo y al Consejo sobre el enfoque global de la UE a los conflictos y crisis exteriores del 11 de diciembre de 2013 (JOIN(2013) 30 final); Dictamen del Comité Europeo de las Regiones: Propuesta de un Nuevo Consenso Europeo sobre Desarrollo: «Nuestro mundo, nuestra dignidad, nuestro futuro» del 30 de junio de 2017 (2017/C 207/08); o las Conclusiones del Consejo sobre el enfoque integrado de los conflictos y crisis exteriores del 22 de enero de 2018 (5413/18).

${ }^{26}$ Estrategia Europea de Seguridad, Una visión común... op. cit, p.22.
} 
La UE cuenta con un importante arsenal de herramientas que pueden implementarse con la finalidad de actuar de forma preventiva, a lo largo de todo el ciclo de vida del conflicto, para alcanzar el enfoque integral. Tradicionalmente, su seña de identidad se ha vertebrado en torno a la política de desarrollo y programas de cooperación, así como ayuda humanitaria y acuerdos comerciales, pues "basta recordar que la Unión Europea es el mayor actor comercial a nivel mundial" (Niño Pérez, 2006, p.136). Al ser el principal proveedor de ayuda al desarrollo, existen importantes ventajas, la Unión tiene un alcance único en muchos países afectados por conflictos, así como el hecho de ser percibido con un bajo perfil político, lo que le convierte en un actor más ventajoso para intervenir de forma preventiva en contextos conflictuales (Schünemann, 2011, p.2). Importantes son también las políticas dirigidas hacia la protección de los derechos humanos y la democracia como factores clave de una agenda de prevención estructural.

Con el paso del tiempo, y sobre todo a finales de los años noventa, la organización empieza a utilizar otras herramientas más centradas en el corto plazo. La diplomacia preventiva puede ser muy útil en la primera etapa del ciclo del conflicto, ya que funciona antes de que se desate la violencia mediante el uso de buenos oficios y negociaciones (Davis, 2018), el diálogo político, el envío de una misión de investigación o un equipo de observadores, el nombramiento de un Representante Especial, las visitas de alto nivel, los enviados especiales, el apoyo a actividades de otros organismos internacionales, el arbitraje, o la puesta en práctica de la diplomacia coercitiva, con "la aplicación de sanciones diplomáticas o la retirada del reconocimiento diplomático" (Marquina y Ruiz, 2005, p.79).

Otros instrumentos para persuadir a las partes involucradas en la crisis de que cambien su comportamiento son los incentivos y las sanciones. Como una especie de medida diplomática coercitiva, las sanciones económicas se utilizan cuando surgen tensiones y hay una crisis manifiesta, cuando la mediación no funciona y antes de considerar una intervención militar. La UE ha impuesto sanciones en varias situaciones, en la mayoría de los casos, en apoyo o implementando las resoluciones del Consejo de Seguridad de Naciones Unidas (Budapest Centre for Mass Atrocities Prevention, 2016, p.83). Según lo acordado por la organización, se pueden utilizar cuando hay "graves violaciones de los derechos humanos o del derecho humanitario" 27 .

${ }^{27}$ Comunicación de la Comisión Europea del 11 de abril de $2001 \ldots$, op. cit. p.24. 
Cuando el conflicto es inminente, el despliegue preventivo de personal civil y militar es una herramienta útil como medida disuasiva. La UE cuenta con misiones civiles y militares con enfoque preventivo, como parte de su Política Común de Seguridad y Defensa (PCSD), y es precisamente lo que le dota de una gran ventaja (Marquina y Ruiz, 2005, p.71-72). Las llamadas misiones Petersberg, que nacieron de la Declaración de Petersberg, adoptada en el Consejo de Ministros de la Unión Europea Occidental (UEO) en junio de 1992, y ampliadas en el Tratado de Lisboa, incluyen misiones de prevención de conflictos ${ }^{28}$.

El uso de la amenaza de la fuerza se refleja en documentos como la Estrategia de Seguridad de 2016, donde se subraya que "las medidas restrictivas [...] son herramientas clave para lograr un cambio pacífico. Pueden desempeñar un papel fundamental en la disuasión, la prevención y la resolución de conflictos" 29 . Añade que "los europeos deben estar preparados para responder rápidamente a las crisis en pleno cumplimiento de la Carta de las Naciones Unidas [...y ] deben desarrollar la capacidad para responder de forma inminente" "30. Sin embargo, "la capacidad de la UE de participar en tareas preventivas coercitivas como la amenaza [del uso de] la fuerza militar [...] es limitada" (Budapest Centre for Mass Atrocities Prevention, 2016, p.66). Por ello, en este sentido, las misiones se han enfocado más en desplegar observadores $o$ en proporcionar apoyo y entrenamiento a las fuerzas locales o apoyo logístico humanitario, y otras tareas como "el desarme, control de armas [...o] acuerdos de no proliferación" (Marquina y Ruiz, 2005, p.78). En este sentido, cuando el ciclo del conflicto se encuentra en la fase de escalada de la violencia, la aplicación de la amenaza del uso de la fuerza puede ser una estrategia ventajosa $y$, aunque la UE la contempla como parte de su política de gestión de crisis, en la práctica no ha sido utilizada tal y como se defiende en la doctrina de prevención de conflictos de la organización, pues se han priorizado elementos civiles antes que otros actores del campo militar (Haastrup, Mah y Duggan, 2021, p.181).

Nos encontramos, por tanto, que la UE dispone de un amplio abanico de medidas, de diferente naturaleza, dirigidas a las distintas fases del conflicto, y respondiendo al enfoque integral que la organización ha

\footnotetext{
${ }^{28}$ Versión Consolidada del Tratado de la Unión Europea, publicado en el Diario Oficial de la Unión Europea el 30 de marzo de 2010 (C 83/15), artículos 21c, 42 y 43.

${ }^{29}$ Estrategia Europea de Seguridad, Una visión común... op . cit., p.28.

${ }^{30}$ Ibídem, p.42.
} 
defendido en la documentación oficial como parte de su doctrina de prevención de conflictos. Sin embargo, si se analiza la documentación en profundidad, pueden detectarse algunos déficits, tanto conceptuales como prácticos, que podrían afectar a la implementación de la prevención en el terreno. En primer lugar, el concepto de prevención de conflictos es, en muchas ocasiones, entendido de forma muy amplia, teniendo como resultado una confusión conceptual con otros términos como diplomacia preventiva $^{31}$ o construcción de la paz (peacebilding) ${ }^{32}$. La ausencia de un marco claro y adaptado a un mundo que cambia de forma vertiginosa sobre lo que la UE entiende por prevención de conflictos afecta a la implementación o despliegue de las medidas que se han mencionado.

En segundo lugar, aunque se puede interpretar que existe una distinción entre las medidas de naturaleza estructural, a largo plazo, y aquellas operativas, para desplegar a corto plazo, lo cierto es que en muchos documentos existe una confusión importante y los límites entre un tipo y otro de medidas no siempre aparecen de forma clara. Además, no existe una doctrina relevante sobre cómo implementar ambos tipos de medidas sobre el terreno, lo cual dificulta la toma de decisiones y perjudica la efectividad de la prevención. Por último, un aspecto a destacar es que, a pesar de la relevancia que la cooperación con otros actores tiene para el éxito de la prevención de conflictos, especialmente el papel de los actores locales, existen aspectos poco claros en este sentido, que se reflejan en el marco de actuación en torno a la autorización, el principio de subsidiariedad, la supremacía, y a otros como la duplicidad de esfuerzos y recursos, o la falta de comunicación entre los diferentes actores que intervienen en una crisis.

${ }^{31}$ Según se interpreta en el este artículo, la diplomacia preventiva es una herramienta dentro del amplio arsenal con el que cuenta la prevención de conflictos.

32 Mientras que algunos académicos y profesionales "hacen todo lo posible para distinguir entre estos conceptos", otros usan los términos indistintamente (Menkhaus, 2004, pp.425-426). La construcción de la paz puede verse como una herramienta para prevenir la violencia ya que sus actividades se enfocan en fortalecer las capacidades de una sociedad para superar sus disputas (OCDE, 1997, p. 9; Naciones Unidas, 2001). Las actividades de prevención de conflictos se pueden implementar en un contexto posconflicto cuando se está desarrollando la consolidación de la paz- con la intención de evitar que un conflicto resurja (Haider, 2014). En resumen, las actividades de consolidación de la paz pueden contribuir de alguna manera a la prevención de conflictos futuros, pero ese no es su objetivo principal. Esta es, desde nuestro punto de vista, la gran diferencia, ya que el objetivo de la prevención de conflictos en esos contextos es evitar que la violencia resurja. 
Por ello, resulta interesante analizar cómo la UE puede impulsar la prevención a través de la cooperación con otros actores regionales, ya que es un aspecto fundamental para mejorar el impacto de sus políticas preventivas en aquellos contextos de crisis. Se ha seleccionado como caso de estudio la CEDEAO, organización que ha puesto en marcha el despliegue preventivo en su área de influencia, así como otras medidas de tipo operativo, a corto plazo, y que mantiene una estrecha relación con Europa.

\section{Fortalecer el PAPEl de los aCtORES Regionales: haCia UN TRABAJO COOPERATIVO}

La UE se encuentra en una posición ventajosa para desplegar políticas preventivas en el continente africano como el proveedor más importante de ayuda oficial para el desarrollo (Schünemann, 2011, p. 2). Mantiene, además, una larga trayectoria de acuerdos con otras organizaciones regionales, apoyando los procesos de integración en otras regiones. Es de destacar las negociaciones que se están llevando a cabo actualmente en el seno de la UE, paralizadas el pasado año por la pandemia, acerca de la renovación, o más bien búsqueda de un mejor sustituto, del ya caduco y tan cuestionado Acuerdo de Cotonú ${ }^{33}$. En el caso concreto de la región de África occidental, la UE inició en 2014 un proceso de acuerdos de asociación económica con la CEDEAO así como con la Unión Económica y Monetaria de África Occidental (UMOA) ${ }^{34}$. Las relaciones se remontan a la década de los años setenta, cuando se funda la CEDEAO, inicialmente focalizadas en apoyar el establecimiento de un mercado común y la creación de una unión aduanera, aunque paulatinamente el campo comercial y económico se fue ampliando a otros como el de la paz y la seguridad (Haastrup, Mah y Duggan, 2021, p.139).

${ }^{33}$ Para ampliar información sobre este Acuerdo así como sobre los pasos que se han dado recientemente, consultar el siguiente enlace de la página del Consejo Europeo: https://www.consilium.europa.eu/en/policies/cotonou-agreement/

34 Acuerdo de Asociación Económica entre los Estados de África Occidental, la Comunidad Económica de los Estados de África Occidental (CEDEAO) y la Unión Económica y Monetaria de África Occidental (UEMOA), de la parte única, y la Unión Europea y sus Estados Miembros, de la otra parte, del 14 de septiembre de 2014 (EU/EPAWA). 
Respecto a su interés por la prevención de conflictos en el continente africano, la Unión

Europea ha adoptado decisiones importantes. En 1994 creó el African Peace Facility, un fondo destinado a financiar a la Unión Africana (UA) para el desarrollo de sus actividades relacionadas con la paz y la seguridad (Schünemann, 2011, p.4). También existe la Asociación Estratégica UE-África (JAES), que se inicia en 2005, asentada en los siguientes pilares: a) Paz y Seguridad; b) Gobernanza Democrática y Derechos Humanos; c) Comercio e Integración Regional; y d) Asuntos Clave de Desarrollo. Dentro de las líneas encuadradas en las políticas de paz y seguridad, la prevención de conflictos ocupa un lugar importante, desde donde se trabaja sobre las causas estructurales de los conflictos y para lo que cuenta con sistemas de alerta temprana.

Otro paso importante en esta materia fue la adopción, en diciembre de 2006, en una reunión del Consejo de Asuntos Generales de la Unión, del Plan para el Fortalecimiento de las Capacidades Africanas para la Prevención, Gestión y Resolución de Conflictos, conocido como Recamp, que pretende dar responsabilidad y titularidad a los actores africanos (Alliot-Marie, 2007). Las relaciones entre la UE y la CEDEAO se desarrollan bajo instrumentos como el European Development Fund (EDF) o el EU Emergency Trust Fund (EUTF), orientadas hacia temáticas como la paz, la seguridad y la estabilidad regional.

Para la UE, el trabajo conjunto en materia de paz y seguridad con los actores locales es una prioridad. Así se recogía ya en el Programa de Gotemburgo. Como recuerdan Haastrup, Mah, y Duggan (2021, p.140), el campo de la paz y seguridad es uno de los prioritarios en las relaciones entre la UE y el continente africano, "debido a los numerosos desafíos de seguridad comunes que afectan a los dos continentes y a la creciente atención que dedica la UE al nexo entre seguridad interior y exterior", y donde la prevención de conflictos aparece como una prioridad para los autores.

En situaciones de crisis, la gestión europea se realiza desde el EEAS, desplegando misiones civiles y militares a través de órganos como el EU Military Staff, desde donde se elabora el Concepto de Operaciones y Plan Operativo, y poniendo en marcha el EEAS Crisis Response System. Esta planificación se hace apoyando a actores locales como la CEDEAO, implicados en los contextos conflictuales sobre los que se van a desplegar las operaciones bajo la CSDP. Algunos ejemplos recientes son las misiones civiles EU Capacity Building Mission (EUCAP Sahel Niger) y 
European Union Capacity Building Mission Mali (EUCAP Sahel Mali) y la militar European Union Training Mission Mali (EUTM Mali) (Haastrup, Mah, y Duggan, 2021, p.84).

\section{1. ¿La CEDEAO como aliada?}

Como ya se ha subrayado, uno de los principales desafíos de la agenda de prevención de conflictos de la UE es impulsar el papel y las capacidades de los actores locales y regionales. En este artículo centraremos la atención en los segundos. Para el éxito de una política europea de prevención de conflictos, la colaboración con esos actores próximos al contexto de crisis es de gran importancia. Los actores regionales han adquirido un papel muy destacado en el campo de la paz y la seguridad en las últimas tres décadas (Berman, 2002; Francis, 2006; Moller, 2009). Este proceso revela una 'regionalización' de la gestión de las crisis y conflictos, la cual ofrece ventajas para fomentar la prevención. Las experiencias de las organizaciones regionales muestran la ventana de posibilidades que ofrece la complementariedad y cooperación entre Estados dentro del complejo regional de seguridad ante una crisis. Tomando como referencia la región de África occidental, se observa cómo en ella conviven una serie de desafíos de seguridad compartidos, lo cual produce una interdependencia de seguridad, ya que las preocupaciones de seguridad nacional de los estados de esa región son muy similares y tan cercanas que no pueden abordarse de forma independiente (Persson, 2012). Así, los problemas regionales se gestionan a través de la cooperación regional (Buzan, Waever \& Wilder, 2003).

El modelo que ha desarrollado la CEDEAO es el ejemplo más interesante para analizar la gestión de los temas de paz y seguridad en el continente africano por parte de una organización regional, donde destacan las medidas destinadas a la prevención de conflictos y alerta temprana (Cuadrado, 2018, p.66). La UE resalta, en la página del EEAS, el potencial de la CEDEAO como promotor de la paz, la seguridad, la democracia y buena gobernanza en la región.

La organización se funda en el año 1975 por medio del Tratado de Lagos, experimentando una reforma importante en 1993. Se presenta como un acuerdo de cooperación económico y comercial para la región de África occidental, sin embargo, pronto empieza a desarrollar una agenda común en materia de seguridad y defensa, que se materializa en el 
Protocolo de No Agresión de 1978 y el Protocolo sobre Asistencia Mutua de 1981, así como la puesta en marcha de su Grupo de Monitorización del Alto el Fuego de la CEDEAO (ECOMOG), en la primera guerra civil de Liberia en 1989.

A finales de la década de 1990, la organización adoptó el Protocolo sobre el Mecanismo de Prevención, Gestión, Resolución, Mantenimiento de la Paz y Seguridad, acordado en la Reunión de Lomé del 10 de diciembre de 1999, que es el marco legal y regulatorio de la CEDEAO en todas las cuestiones relacionadas con la paz y la seguridad ${ }^{35}$ (CEDEAO, 2018 , p.16). Este instrumento demuestra que la prevención de conflictos es de gran importancia para la organización, ya que, entre sus los objetivos, se encuentran la prevención de: conflictos internos e interestatales; graves violaciones de los derechos humanos; o un derrocamiento o intento de derrocamiento de un gobierno elegido democráticamente.

Según el citado Protocolo, el órgano central para cuestiones de paz y seguridad es el Consejo de Mediación y Seguridad, que puede actuar en las circunstancias que hemos mencionado. El resto de órganos que participan en esta estructura de paz y seguridad son: el Consejo de Sabios, integrado por personalidades eminentes que actúan como mediadores; el ECOMOG; o la brigada ECOBRIG, ECOWAS Reserve Force, que es una de las cinco brigadas de la Fuerza Africana de Reserva de la Unión Africana, una fuerza continental de mantenimiento de la paz formada "por componentes militares, policiales y civiles, que se encuentran en reserva en las regiones de origen y dispuestos a actuar en nombre de la Unión Africana en tiempos de crisis" (Institute for Security Studies, 2015). Esta fuerza se puede utilizar, por ejemplo, para un despliegue preventiva.

El siguiente paso en la estructura de paz y seguridad de la CEDEAO fue la aprobación del Protocolo sobre Democracia y Buen Gobierno, adoptado el 21 de diciembre de 2001 y firmado en Dakar, enfocado a gestionar aspectos estructurales como injusticias o mal gobierno, entre otros, manifestando la necesidad de incorporar disposiciones sobre temas como la prevención de crisis internas.

35 Documento de la Comisión de la CEDEAO de febrero de 2018 sobre Directrices para la Mediación., p.16. Disponible en: https://ecpf.ecowas.int/wpcontent/uploads/2016/01/ECOWAS-ENGLISH-230518.pdf 
El acuerdo más reciente sobre políticas preventivas fue el Marco de Prevención de Conflictos (ECPF por sus siglas en inglés) de la CEDEAO, adoptado por el Consejo de Mediación y Seguridad en enero de $2008^{36}$. Establece varios componentes, desde la alerta temprana y la diplomacia preventiva, la democracia y gobernanza política, los derechos humanos, la gobernanza de los recursos naturales, el desarme, las cuestiones de género, el empoderamiento de la juventud, o la asistencia humanitaria. Esto demuestra que la CEDEAO comprende la prevención de conflictos desde un enfoque integral, como así hace también la UE. Este Marco buscaba ser el impulso definitivo para la prevención de conflictos, actuando como marco estratégico para la seguridad humana y la prevención y adoptando una postura más proactiva y operativa de prevención de conflictos en los Estados miembros.

Finalmente, como resultado de los acuerdos alcanzados tras el lanzamiento del Marco, en 2015 se creó la División de Facilitación de la Mediación (MFD) de la CEDEAO con el objetivo de promover la diplomacia preventiva en la región a través del apoyo operativo, con el establecimiento de un centro de recursos de mediación para garantizar el desarrollo de capacidades en la mediación.

Bajo este marco regulatorio que ha evolucionado y se ha adaptado a los cambios y necesidades del escenario de seguridad de la región, la CEDEAO ha desarrollado una importante actividad en diplomacia preventiva y mediación en diferentes contextos (Cuadrado, 2019).

\subsection{Obstáculos por superar}

La implicación de las organizaciones regionales en temas securitarios tiene importantes ventajas, entre las que se encuentra un mejor conocimiento del contexto y, por lo tanto, de las raíces de los conflictos. Las organizaciones regionales están bien posicionadas para detectar señales de alerta temprana de un conflicto inminente. Son actores que conocen mejor el terreno para comprender la situación desde otra perspectiva y tienen un interés directo en las repercusiones y soluciones del conflicto. Disfrutan (aunque no siempre) de una mayor legitimidad popular en las zonas de conflicto que los mediadores internacionales, como pueden ser las instituciones europeas, y las fuerzas

\footnotetext{
36 Marco para la Prevención de Conflictos de la CEDEAO (Reglamento MSC/REG.1/01/08).
} 
de mantenimiento de la paz (Gowan, 2017). Como resultado, los actores regionales, con el apoyo de la comunidad internacional, juegan un papel de liderazgo en la prevención de conflictos.

Por ello, la UE puede y debe apoyar y apoyarse en estos actores para impulsar la agenda de prevención de conflictos, especialmente en espacios geográficos de especial relevancia para Europa como es África occidental. Existe, así, una relación mutuamente beneficiosa, ya que son los actores regionales (y locales) los que poseen un mejor conocimiento sobre el contexto conflictual y las dinámicas, pero dependen, en gran medida, de un apoyo logístico, material y humano del exterior, donde los fondos aportados por las instituciones y organismos europeos son fundamentales. La implementación de políticas de prevención de conflictos en la región de África occidental se ve afectada por la falta de financiación, que proviene de los Estados Miembros. Los limitados recursos humanos y financieros afectan al desarrollo de las medidas y obligan a la organización regional, la CEDEAO, a elegir en qué contextos o situaciones intervenir. Así, en una región donde existen importantes desafíos para la paz y la seguridad, la falta de recursos hace que algunos factores de riesgo no reciban suficiente atención y las acciones se enfoquen en las amenazas más inmediatas. Por tanto, los actores regionales como la CEDEAO dependen de actores externos, como la UE, lo que hace que el éxito de la regionalización de la seguridad se vea limitado.

Esa dependencia de actores externos es una de las razones que impulsa la participación de estos en la crisis, lo que provoca que se realicen diversas acciones e intervenciones, a diferentes niveles, al mismo tiempo, con "superposición institucional de actores" (Sousa, 2017, p. 572) que genera confusión y dificulta la intervención o la actuación preventiva. Esta situación se ha vivido con frecuencia en la región de África occidental, donde la falta de coordinación entre los diferentes actores que participan en contextos conflictuales puede dificultar la gestión: desde países de la región, organismos regionales de la CEDEAO, hasta actores externos como la Unión Africana, Naciones Unidas o la UE, pero también países europeos de forma bilateral, como es el caso destacado de Francia. Así ocurrió, por ejemplo, en la crisis de Malí, cuando se solicitó la intervención militar francesa para recibir asistencia en la batalla contra los insurgentes en el norte del país, o en Gambia, cuando la Comisión de la CEDEAO declaró que no dudaría en buscar apoyo financiero y logístico de organismos como la Unión 
Europea para gestionar la crisis (Haastrup, Mah, y Duggan, 2021, p.257). Como señaló Williams haciendo mención a Naciones Unidas (2017, p.129), es necesario que organizaciones como esa o como la UE desarrollen "mecanismos de apoyo adecuados" a las organizaciones regionales en África, especialmente por la falta de "financiamiento sostenible y flexible" que tienen.

Las operaciones iniciadas por organizaciones regionales necesitan la autorización del Consejo de Seguridad antes de su aplicación (Carta de las Naciones Unidas, Capítulo VIII, Art. 53), lo que a menudo provoca "desacuerdos [...] en varios niveles de adopción de decisiones" (Desmidt y Hauck, 2017, p.15). En el caso de la CEDEAO, "no reconoce explícitamente la supremacía del CSNU [Consejo de Seguridad de las Naciones Unidas] en términos de paz y seguridad" (Jetschke y Schlipphak, 2019, p. 4). Al mismo tiempo, existe un desacuerdo entre la UA y las organizaciones regionales, como la CEDEAO, donde una "falta de claridad sobre la subsidiariedad y la división del trabajo [...] a menudo conduce a soluciones ad-hoc y pragmáticas" (Desmidt, 2019, p. 2), afectando a la implementación de medidas preventivas. Desde la UA, se plantea el concepto de primacía, sin embargo, el principio de subsidiariedad "establece que las respuestas al conflicto deben ser formuladas por organizaciones con mayor proximidad" (Desmidt, 2019, p. 12). Esta falta de acuerdo e interpretación del marco normativo de los actores africanos también afecta a las relaciones con la UE y la toma de decisiones ante una crisis. Bajo ese marco confuso, los actores externos, como la organización europea, pueden terminar estableciendo relaciones duplicadas, mandatos contradictorios y solapados que respondan a marcos normativos diferentes y poner en marcha misiones en unos contextos y no en otros de igual naturaleza y emergencia. Así, como señalan Nathan et. al (2017), los documentos de política son ambiguos o contradictorios cuando se trata de la gestión de crisis. Este hecho provoca superposición y confusión entre todos los actores que intervienen en el contexto, dificultando la capacidad de acción para intervenir o actuar de manera preventiva.

La falta de claridad en los mandatos de cada organización, las confusiones derivadas de conceptos como la autorización, la primacía o la subsidiariedad, tienen como resultado la duplicidad de esfuerzos y recursos, la falta de comunicación e incluso la paralización de la toma de decisiones en algunas ocasiones. 
Estos problemas no solo se dan en torno a las relaciones entre organizaciones, sino en el seno de la propia UE. A la hora de poner en marcha la agenda de gestión de crisis, en la toma de decisiones se ven implicados, en mayor o menor medida, distintos órganos (Comisión Europea, Consejo Europeo, Estados Miembros, Parlamento Europeo), con visiones, parámetros y formas de proceder diferentes. La dificultad principal recae en poner en común las prácticas de "las instancias comunitarias y las de los Estados Miembros" (Niño Pérez, 2006, p.142). Dentro del abanico de medidas preventivas, la toma de decisiones puede proceder desde el Consejo Europeo, la Comisión, el EEAS o varias agencias europeas, por lo que los criterios de actuación son diferentes, a lo que añadir el papel que de forma bilateral pueden jugar algunos Estados Miembros.

La complejidad institucional tiene repercusiones a la hora de implementar las políticas de prevención, pues la toma de decisiones en esta materia está envuelta por un complejo marco legal que, en ocasiones, es contradictorio. Como se subraya en un informe reciente (Particip $\mathrm{GmbH}$ et. al, 2020), no pueden identificar en la UE de forma clara aquellos actores específicos responsables de la implementación de los compromisos adoptados, hace veinte años, en el Programa de Gotemburgo.

\section{REFLEXIONES FINALES}

En este artículo de diagnóstico sobre la política europea de prevención de conflictos, se ha querido resaltar el potencial que esta tiene para el contexto actual de seguridad internacional, donde la UE cuenta con un amplio abanico de medidas así como con una posición ventajosa. Un factor fundamental para impulsar la agenda preventiva europea y hacerla más eficaz y adaptada al contexto de cada crisis es el apoyo a los actores regionales; reforzar las relaciones y el trabajo cooperativo. Esta colaboración puede traducirse en un marco mutuamente beneficios pues, mientras la UE tiene la experiencia en misiones internacionales y dispone de los recursos necesarios para abordar crisis cada vez más complejas, los actores locales y/o regionales cuentan con el conocimiento sobre el contexto conflictual y los elementos subyacentes al mismo.

La organización regional de África occidental, la CEDEAO, es un ejemplo claro: por un lado, simboliza una zona de importancia para la UE, por lo que existe un interés en desarrollar políticas preventivas que 
mejoren la inestabilidad presente $y$, por otro lado, la organización africana cuenta con experiencia en la gestión de crisis con un enfoque preventivo en su área de influencia. En el complejo regional de seguridad que engloba la CEDEAO se desencadenan crisis y conflictos que, en ocasiones, reciben el interés de las instituciones europeas, poniendo en marcha diferentes medidas, a corto y largo plazo. Cuando eso ocurre, se produce una configuración de diferentes actores que intervienen en el contexto, ya sea de forma unilateral o colaborativa, secuencialmente o con un solapamiento de mandatos y misiones. Esa multiplicidad de actores, que pueden tener diferentes objetivos y doctrinas, suponen un desafío para la eficacia de las políticas de prevención.

Para poder implementar un trabajo cooperativo entre la UE y actores clave para la gestión de crisis y la prevención, como es la CEDEAO, es necesario, primero, aclarar aspectos que afectan a la toma de decisiones e implementación de medidas dentro de la UE, como la precisión de conceptos, la mejor definición de los órganos que intervienen y delimitar el papel de cada uno, así como definir y desarrollar mejor el tipo de medidas y herramientas que pueden desplegarse. En segundo lugar, es necesario resolver los problemas derivados de las intervenciones multiactorales para mejorar la efectividad de la coordinación sobre el terreno, aclarar el papel de cada actor y los tiempos de intervención, para evitar duplicidad de esfuerzos y recursos, y trabajar para definir un marco común sobre la doctrina de prevención de conflictos. Estos elementos son fundamentales para impulsar y hacer efectiva una política europea preventiva y será un paso más para que la UE mantenga o recupere su papel como actor global en la gestión de crisis de su vecindad más próxima para el futuro.

El objetivo de este artículo ha sido dibujar una aproximación a las posibilidades que existen en la cooperación y trabajo conjunto entre dos organizaciones, la UE y la CEDEAO, en materia de prevención de conflictos, teniendo en cuenta que esta política debe ser una prioridad para ambas. Debido al papel relevante que ocupa y que tendrá en el futuro la agenda preventiva, el siguiente paso de esta investigación es evaluar la implementación de la agenda de la UE sobre el terreno y el desarrollo del trabajo conjunto con la CEDEAO, con sus luces y sus sombras. Por ello, la autora considera de gran importancia continuar la investigación haciendo una selección de casos en la región, como la crisis de Malí, Gambia y Guinea-Bissau, para conocer cómo la teoría se lleva 
sobre el terreno e identificar de forma más clara los obstáculos que afectan a la implementación de la agenda preventiva de la UE.

\section{Referencias bibliográficas}

Alliot-Marie, M. (2007): "For the EU, Africa's ills must be dealt with by Africans, Consejo Europeo del 6 de julio de 2007. Disponible en: http://www.consilium.europa.eu/ueDocs/cms_Data/docs/pressData/e n/esdp/95218.pdf

Berman, E. G. (2002): “African Regional Organisation' Peace Operations. Developments and Challenges". African Security Review 11(4), 33-44.

Budapest Centre for Mass Atrocities Prevention (2016): "African Regional Communities and the Prevention of Mass Atrocities". African Task Force on the Prevention of Mass Atrocities. Disponible en: $\quad$ http://www.genocideprevention.eu/wpcontent/uploads/2016/10/African-Regional-Communities-and-thePrevention-of-MassAtrocities-Final-Report-African-Task-Force.pdf

Buzan, B., Wæver, O., y Wilde, J. (2003): Regions and Powers: The Structure of International Society. Cambridge: Cambridge University Press.

Carnegie Corporation of New York (1997): Preventing Deadly Conflict. Informe final. Nueva York: Carnegie Commission on Preventing Deadly Conflicts.

Center for Systemic Peace (2019): Center for Systemic Peace, Major Episodes of PoliticalViolence, 1946-2018 (War List). Disponible en: https://www.systemicpeace.org/inscrdata.html

Cuadrado, J. (2018): Los sistemas de alerta temprana en la prevención de conflictos armados. Un estudio comparado en Africa occidental. Universidad Nacional de Educación a Distancia, tesis doctoral. Disponible en: http://e-spacio.uned.es/fez/eserv/tesisuned:ED-Pg$\underline{\text { SegInt-Jcuadrado/CUADRADO_BOLANOS Jara Tesis.pdf }}$ 
Davis, L. (2018): "Betwixt and between: conceptual and practical challenges of preventing violent conflict through EU external action". Global Affairs 4(2-3), 157-169.

Desmidt, S. y Hauck, V. (2017): "Conflict management under the African Peace and Security Architecture (APSA). Analysis of conflict prevention and conflict resolution interventions by the African Union and Regional Economic Communities in violent conflicts in Africa for the years 2013-2015". European Centre for Development Policy Management, Discussion Paper 211. Disponible en: https://ecdpm.org/publications/conflict-management-under-theapsa/

Desmidt, S. (2019): "Conflict management and prevention under the African Peace and Security Architecture (APSA) of the African Union". Africa Journal of Management. African Journal of Management 5(1), 79-97.

Francis, D. J. (2006): "Linking peace, security and developmental regionalism: Regionaleconomic and security integration in Africa". Journal of Peacebuilding \& Development 2(3), 7-20.

Gowan, R. (2017): "Bordering on Crisis: Europe, Africa, and a New Approach to Crisis Management". European Council on Foreign Relations, Policy Brief. Disponible en: https://www.ecfr.eu/page//Bordering_on_crisis02.pdf

Haastrup, T., Mah, L. y Duggan, N. (2021): The Routledge Handbook of EU-Africa Relations. Nueva York: Routledge.

Institute for Security Studies (2015): "Understanding the African Standby Force, rapid deployment and Amani Africa II". ISS Media Toolkit. Disponible en:

https://issafrica.s3.amazonaws.com/site/uploads/4-11-2016-ASFAmani-Media-Toolkit1.pdf

Jetschke, A. y Schlipphak, B. (2019): "MILINDA: A new dataset on United Nations-led and non-united Nations-led peace operations". Conflict Management and Peace Science 37(5), 605-629.

Juncos, A. y Blockmans, S. (2018): “The EU's role in conflict prevention and peacebuilding: four key challenges". Global Affairs 4(2-3), 131140. 
Marc, A., Verjee, N. y Mogaka, S. (2015): The Challenge of Stability and Security in West Africa. Washington: Banco Mundial.

Marquina, A. y Ruiz, X. (2005): “A European competitive advantage? Civilian instruments for conflict prevention and crisis management". Journal of Transatlantic Studies 3(1), 71-87.

Menkaus, K. (2004): "Conflict prevention and human security: issues and challenges. Conflict, Security \& Development 4(3), 419-463.

Moller, B. (2009): "The African Union as Security Actor: African Solutions to African Problems". Working Paper 2, Crisis State Research Centre. Disponible en: http://eprints.1se.ac.uk/28485/1/WP57.2Moller.AU.pdf

Newman, E. (2009): “Conflict Research and the 'Decline' of Civil War". Civil Wars, 11(3), 255-278.

Nathan, L., Day, A., Honwana, J, y Brubaker, R. (2018): “Capturing UN Preventive Diplomacy Success: How and Why Does It Work?". United Nations University Centre for Policy Research. Disponible en: file:///C:/Users/Daniel/Downloads/Nathan\%20et\%20al.\%20UNPreventive-Diplomacy-Policy-Paper-and-Case-Studies.pdf

Niño Pérez, J. (2006): "La política de prevención de conflictos de la Unión Europea tras el 11 de eptiembre de 2001". Cuadernos Europeos de Deusto, 34, 129-148.

Particip GmbH, Ecorys, ECDPM, Fiscus, Itad y OPM (2020): “External Evaluation of EU's Support to Conflict Prevention and Peacebuilding (CPPB) 2013-2018". Informe elaborado por encargo de la Comisión Europea. Disponible en: https://ec.europa.eu/international-partnerships/system/files/cppbeval-final-report-2020-vol-1_en.pdf

Persson, J. (2012): Regions as Security Providers. The Evolution of the West African Regional Security Complex. Universidad de Lund, tesis doctoral. Disponible en: https://pdfs.semanticscholar.org/cae5/663f43e48a7899b2f04c9e2ec5 6a60bb6aca.pdf

Ramsbotham, O., Miall T. y Woodhouse, H. (2011): Resolución de conflictos. La prevención, gestión y transformación de conflictos letales. Barcelona: Bellaterra. 
Sousa, R. (2017): "United Nations Security Council primacy over military interventions in Africa and the African Peace and Security Architecture (APSA)". En Moita, L. y Pinto, L. V. (coord.), Espaços económicos e espaços de segurança. Lisboa: UAL y OBSERVARE, pp. 519-572.

Stewart, E. (2006): The European Union and Conflict Prevention: Policy Evolution and Outcome. Berlín: Lit Verlag.

Williams, P. (2017): "Global and Regional Peacekeepers: Trends, Opportunities, Risks and a Way Ahead". Global Policy 8(1), 124129.

Wouters, J. y Naert, F. (2003): "The EU and conflict prevention: a brief historic overview". Working Paper n. 52, Instituto de Derecho Internacional, Universidad de Leiden. Disponible en: https://www.law.kuleuven.be/iir/nl/onderzoek/workingpapers/WP52e.pdf 